\title{
A REMARK ON SIMPLY-CONNECTED 3-MANIFOLDS
}

\author{
BY V. POÉNARU
}

Communicated by William Browder, May 6, 1974

In this note we will describe an "infinite process", related to "wild topology", with applications to closed, smooth manifolds.

DEFINITION. Consider an increasing sequence of solid tori:

$$
T_{1} \hookrightarrow T_{2} \hookrightarrow \cdots \hookrightarrow T_{n} \hookrightarrow T_{n+1} \hookrightarrow \cdots
$$

$\left(T_{i}=k(i) \#\left(S^{1} \times D^{2}\right)\right)$ such that:

(a) $T_{k} \subset$ int $T_{k+1}$, and $T_{k}$ is a smooth submanifold of $T_{k+1}$.

(b) The natural inclusion $T_{k} \rightarrow_{j_{k}} T_{k+1}$ is null-homotopic.

The open 3-manifold $W=\underline{\lim } T_{i}$ will be called a Whitehead manifold. It is an easy (and well-known) exercise to show that for any Whitehead manifold $W$, one has: $W \times R=R^{4}$.

Theorem 1. Let $X$ be a smooth 3-manifold with $\pi_{1} X=0, T$ a solid torus, and $T \rightarrow_{j} X$ a smooth embedding. There exists a Whitehead manifold $W$ defined by a sequence of nested tori:

$$
T=T_{1} \stackrel{j_{1}}{\longrightarrow} T_{2} \stackrel{j_{2}}{\longrightarrow} \cdots
$$

and a smooth embedding $W \rightarrow_{k} X$ such that the following diagram is commutative:

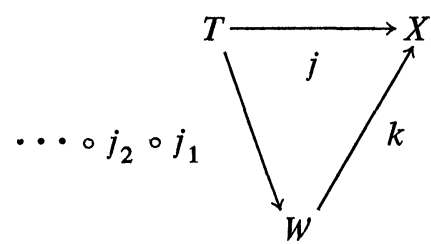

Proof. It suffices to show that there exists a solid torus $T_{2}$ and a commutative diagram of smooth embeddings:

AMS (MOS) subject classifications (1970). Primary 22-XX. 


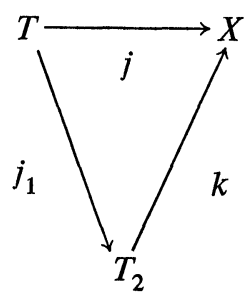

such that $j_{1}$ is null-homotopic. (Afterwards we can continue this process indefinitely.)

Consider a wedge (bouquet) of circles $K=\bigvee_{i=1}^{p} S_{i}^{1}$ which is a spine of $T$. There exists a commutative diagram:

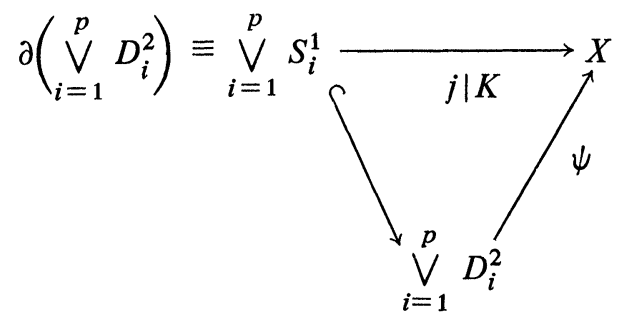

where $\psi$ is a generic immersion, without triple points, such that the set of double points is a union of (disjoined) arcs.

Take $T_{2}=$ a regular neighborhood of $\psi\left(\bigvee_{i=1}^{p} D_{i}^{2}\right)$ in $X$ (containing $T$ in its interior) a.s.o.

COROLlARY 2. Let $\Sigma^{3}$ be a smooth homotopy 3-sphere. There exist two open subsets $U_{1}, U_{2} \subset \Sigma^{3} \times R$ such that:

(a) $\Sigma^{3} \times R=U_{1} \cup U_{2}$,

(b) $U_{i}$ is diffeomorphic to $R^{4}$.

Proof. Let $\Sigma^{3}=T^{\prime} \cup T^{\prime \prime}$ be a Heegaard decomposition, and consider the Whitehead manifolds $W^{\prime}, W^{\prime \prime}$ (containing $T^{\prime}, T^{\prime \prime}$ and contained in $\Sigma^{3}$ ) provided by Theorem 1 .

Since $W \times R=R^{4}$ for any Whitehead manifold, we can take $U_{1}=$ $W^{\prime} \times R \subset \Sigma^{3} \times R, U_{2}=W^{\prime \prime} \times R \subset \Sigma^{3} \times R$. Q.E.D.

DÉPARTEMENT DE MATHÉMATIQUES, UNIVERSITÉ PARIS XI, 91-ORSAY, FRANCE 